\title{
Prior cognitive activity implicitly modulates subsequent emotional responses to subliminally presented emotional stimuli
}

\author{
Saea Iida • Takashi Nakao • Hideki Ohira
}

Published online: 29 February 2012

(C) Psychonomic Society, Inc. 2012

\begin{abstract}
It has been reported that engagement in several kinds of cognitive activity can successfully inhibit unpleasant emotions. In this study, we tried to replicate the previous finding that cognitive activity can modulate subsequent psychological and physiological emotional processes and to investigate whether prior cognitive activity can attenuate implicit emotional processes triggered by subliminal emotional stimuli. Sixty students were randomly divided into three groups (cognitive task group, noncognitive task group, control group). The cognitive task group was asked to engage in an $n$-back task, while the control group was asked to stay calm. The noncognitive task group was asked to do a handgrip-squeezing task. All participants then engaged in a version of a subliminal affective priming task where they were unconsciously exposed to affectively negative pictures. The cognitive task group showed lower negative experiences after the subliminal affective priming task and a substantial reduction in their heart rate responses, as compared with the other
\end{abstract}

S. Iida $\cdot$ H. Ohira $(\bowtie)$

Department of Psychology, Graduate School of Environmental

Studies, Nagoya University,

Furo-cho, Chikusa-ku,

Nagoya, Aichi 464-8601, Japan

e-mail: ohira@lit.nagoya-u.ac.jp

T. Nakao

Mind, Brain Imaging and Neuroethics Institute of Mental Health Research, Royal Ottawa Health Care Group, University of Ottawa, Ottawa, Canada

S. Iida $(\square)$

Institute for Developmental Research,

Aichi Human Service Center,

713-8 Kamiya-cho,

Kasugai, Aichi 480-0392, Japan

e-mail: saea7@yahoo.co.jp groups. These results provide evidence that engagement in cognitive activity can attenuate emotional processes in an automatic and unconscious manner.

Keywords Cognitive activity · Emotion · IAPS .

Subliminal affective priming task

\section{Introduction}

It has been reported that a wide range of emotion regulation strategies that rely on cognitive activity are effective for inhibition of unpleasant emotions (e.g., Erk, Abler, \& Walter, 2006; Hariri, Mattay, Tessitore, Fera, \& Weinberger, 2003; Liberzon et al., 2000; Monk et al., 2003). In line with such previous findings, we (Iida, Nakao, \& Ohira, 2011) showed that engagement in several cognitive tasks for even $5 \mathrm{~min}$ implicitly attenuated subsequent unpleasant emotional responses triggered by affectively negative pictures. Such an attenuating effect did not appear after a noncognitive task, such as a handgrip-squeezing task. However, it is still unclear how cognitive activity modulated subsequent emotional processes.

Emotional processes contain bottom-up appraisal of stimuli and top-down reappraisal of them (Ochsner et al., 2004). Bottom-up appraisal consists of the emotional responses to inherently emotional perceptual properties of the stimuli, while top-down reappraisal acts on the emotional responses by cognitive evaluations (McRae, Misra, Prasad, Pereira, \& Gross, 2011). The top-down process requires consciousness and the awareness of emotions (Ochsner \& Gross, 2007) and is linked to current intentions, plans, and goals (goal based). On the other hand, bottom-up appraisal is more stimulus based, relying on sensory system information. It is of great interest whether bottom-up appraisal or top-down reappraisal 
is a more appropriate explanation for the implicit attenuation effects of negative emotion by prior cognitive activity. However, in generation of an emotion, processes of both bottom-up appraisal and top-down reappraisal occur in parallel in a very short time (Barrett, Ochsner, \& Gross, 2007), and thus it is somewhat difficult to dissociate the effects of prior cognitive activity on these two processes in conventional experimental paradigms of emotion regulation.

In this study, as a first step to elucidate the underlying mechanisms of the effects of prior cognitive tasks in attenuating negative emotions, we specifically focused on the bottom-up process. To exclude involvement of the topdown process, we used a procedure of subliminal affective priming (e.g., Jostmann, Koole, Van der Wulp, \& Fockenberg, 2005; Wallbott \& Ricci-Bitti, 1993), where participants are first exposed to a positive or negative affective stimulus (prime) for very brief durations that preclude conscious detection, followed by an affectively neutral stimulus (target). In such a situation, participants' affective reactions to the neutral target are assessed (for recent reviews, see Berridge \& Winkielman, 2003; Zajonc, 2000). Typically, participants' emotional reactions are unconsciously shifted to the direction congruent with the affective valence of the prime stimulus (subliminal affective priming effect). We used negative and neutral affective pictures for the prime stimuli, in line with Iida et al. (2011), and we used neutral and novel figures for the target stimuli. A prevailing theory has suggested that subliminal affective reactions occur in a very short time and, thus, are unimpeded by top-down processes (Zajonc, 2000). If prior performance of a cognitive task can attenuate subsequent bottom-up processes of an emotion, the subliminal affective priming effect will be diminished or disappear. To examine this hypothesis, the effects of prior cognitive activity on subjective evaluation of the neutral targets that were preceded by affectively negative primes were assessed. Additionally, if prior performance of a cognitive task can attenuate subsequent bottom-up processes of an emotion, implicit emotional responses caused by a subliminal prime stimulus will also be diminished or disappear. For a test of this hypothesis, on the basis of our previous finding that prior cognitive tasks reduced both subjective emotional experiences and physiological responses to emotional stimuli (Iida et al., 2011), we measured both self-reports and a physiological index — namely, heart rate (HR) - as emotional responses.

\section{Method}

Participants

Sixty healthy graduate and undergraduate students who had no history of major neurological or psychiatric disorders volunteered to take part in the study. Each participant gave informed consent. They were randomly divided into three groups (cognitive task group, noncognitive task group, and control group). Each group included 16 males and 4 females. The average age of the cognitive task group was 24.3 years (range, 21-27; $S D=2.0$ ), that of the noncognitive task group was 26.5 years (range, 22-31; $S D=2.9$ ), and that of the control group was 25.7 years (range, 21-29; $S D=2.6$ ).

\section{Procedure}

After an instruction about the procedure of the experiment, physiological sensors were attached to participants. The participants underwent four testing stages within a single experimental session: baseline $(5 \mathrm{~min})$, cognitive task (5 min), emotional task (10 min), and recovery $(5 \mathrm{~min})$. In the baseline stage, participants were asked to stay calm. The cognitive and noncognitive task groups were then asked to perform their respective tasks, and the control group was again asked to stay calm. After the cognitive task stage, all of the participants conducted the emotional task. In the recovery stage, the participants were again asked to stay calm. We collected data of participants' self-reports about their unpleasant emotions after each stage and measured HR responses throughout the entire session.

\section{Cognitive task}

A sequence of one-digit numbers were presented in a random order. Each number was shown for $1 \mathrm{~s}$ after an asterisk (fixation point) that was presented at the center of a screen for $1 \mathrm{~s}$. The cognitive task group was asked to engage in a 2-back task; that is, they had to press a "target key" when the number presented was identical to the first of the two previous numbers. Otherwise, they were to press a "nontarget key." This response was conducted by using an RB-730 response pad (Cedrus Corporation, San Pedro, CA). Iida et al. (2011) examined the effects of prior cognitive task performance on subsequent emotional responses, using three typical executive function tasks- the $n$-back task, the go/no-go task, and a modified version of the Wisconsin cardsorting test (WCST) - all of which require different cognitive abilities. The $n$-back task requires maintenance and permanent update of relevant pieces of information in working memory, the go/no-go task requires inhibitory control of a response, and the WCST requires set shifting. Engagement in these three tasks equally attenuated subsequent emotional responses, regardless of their cognitive natures. Hence, in this study, as a typical cognitive task, we used the 2-back task, which is frequently used as a measure of 
executive function (e.g., Baddeley, 2003). All participants performed this task at correct rates of $82 \%-96 \%$.

\section{Noncognitive task}

The noncognitive task group was instructed to squeeze a 15$\mathrm{kg}$ handgrip commonly used to train hand muscles. The handgrip consists of two handles connected by a wound spring and is designed to build muscles in the forearm. Individuals grasp the handgrip, which brings the handles together and compresses the spring, thereby creating resistance. Participants were asked to squeeze the handgrip once every $2 \mathrm{~s}$ for $5 \mathrm{~min}$. Iida et al. (2011) showed that this task requires a similar degree of effort to the cognitive tasks that were used in the study. Thus, we used the same handgrip-squeezing task as a noncognitive task in this study and evaluated the degree of effort in the same way as in Iida et al.

\section{Emotional task}

For prime stimuli, 60 unpleasant pictures and 60 neutral pictures selected from the International Affective Picture System (IAPS; see the Appendix; Lang, Bradley, \& Cuthbert, 1999) were used in the emotional task. In our preliminary study where 13 graduate and undergraduate students who did not participate in the present experiment rated the 658 pictures in dimensions of valence, arousal, and visual complexity, the two categories of pictures produced a significant difference in valence $[M=3.2$ and 5.8 for unpleasant and neutral pictures, respectively, on a 9-point scale (1, unpleasant; 9, pleasant); $t(118)=6.56, p<.01, r=.23]$. There was no significant difference in arousal levels between the two categories [6.0 and 5.6 for unpleasant and neutral pictures, respectively, on a 9-point scale (1, calm; 9, excited); t(118) = 1.64, n.s., $r=.14$ ]. Additionally, ratings of visual complexity showed no difference between the two categories of pictures [6.8 and 6.7 for unpleasant and neutral pictures, respectively, on a 9-point scale $(1$, simple; 9 , complex $) ; t(118)=1.38$, n.s.,$r=.09$ ].

For target stimuli, 60 novel figures were selected from the set of novel shapes, which were validated by evaluation of levels of verbalization, association, and simplicity (Endo, Saiki, \& Saito, 2001). Each target stimulus was used twice, paired with a negative prime and a neutral prime. Combinations of a target stimulus, a negative prime, and a neutral prime were randomized.

On each trial, participants first gazed at a fixation point (a black and then a red cross) for 1,900 ms. To prevent participants from missing a prime stimulus, we asked participants to concentrate on the red cross when it appeared. Then a prime stimulus was presented for $15 \mathrm{~ms}$. It was followed by a masking stimulus presented for $85 \mathrm{~ms}$ and then by a target stimulus presented for $1,000 \mathrm{~ms}$. Finally, participants evaluated the affective negativity both of the target stimulus and of their feelings on 5-point scales. Then the next trial began. Participants performed 120 trials in total (see Fig. 1). The order of trials where the negative primes were presented and trials where the neutral primes were presented was randomized. Participants responded by using an RB-730 response pad (Cedrus Corporation).

\section{Manipulation check}

To check awareness of the prime stimuli, we used a forced choice test after the termination of the recovery stage. First, participants gazed at a fixation point (the black cross followed by the red cross) for $1,900 \mathrm{~ms}$. Then a prime (negative or neutral) that had been presented in the emotional task was presented for $15 \mathrm{~ms}$ and immediately followed by a masking stimulus presented for $85 \mathrm{~ms}$. After the masking stimulus disappeared, two pictures were displayed at lateral positions on the screen for $2 \mathrm{~s}$ : an image of the actual prime on one side of the screen and a new image that had not been presented (a distractor) on the other side of the screen. Participants were asked to infer and to indicate which image was the prime by pressing a corresponding button on a response box. The screen position (right, left) of the prime image was randomized. The 120 prime stimuli used in the emotional task were tested.

\section{Measures}

Self-reports Unpleasant emotions induced by the emotional task were measured by a negative affect scale of the Positive and Negative Affect Schedule (PANAS, a Japanese version; Sato \& Yasuda, 2001). The negative affect scale included eight items, which were rated by 5-point scales. The PANAS scores were recorded after each stage of the experiment. On each trial in the emotional task, a participant evaluated affective negativity for a target image and for his/her current feeling. The evaluation scores were averaged for each condition of prime (negative, neutral) within each participant for statistical analyses. To measure and match the task load of the cognitive task and the noncognitive task, we used a Japanese version of the NASA Task Load Index (Haga \& Mizukami, 1996), which was used in our previous study (Iida et al., 2011). The NASA Task Load Index includes six dimensions: mental demand, physical demand, temporal demand, performance, effort, and frustration. The use of these six subscales to compute an overall workload score has been found to reduce variability among participants, relative to a one-dimensional workload scale (Hart \& Staveland, 1988). Twenty-step bipolar scales are used to obtain ratings for these dimensions. A score from 0 to 100 (assigned to the nearest 5) is obtained on each scale. The mean scores for each subscale were calculated. An overall workload 
Fig. 1 Time lime of emotional task

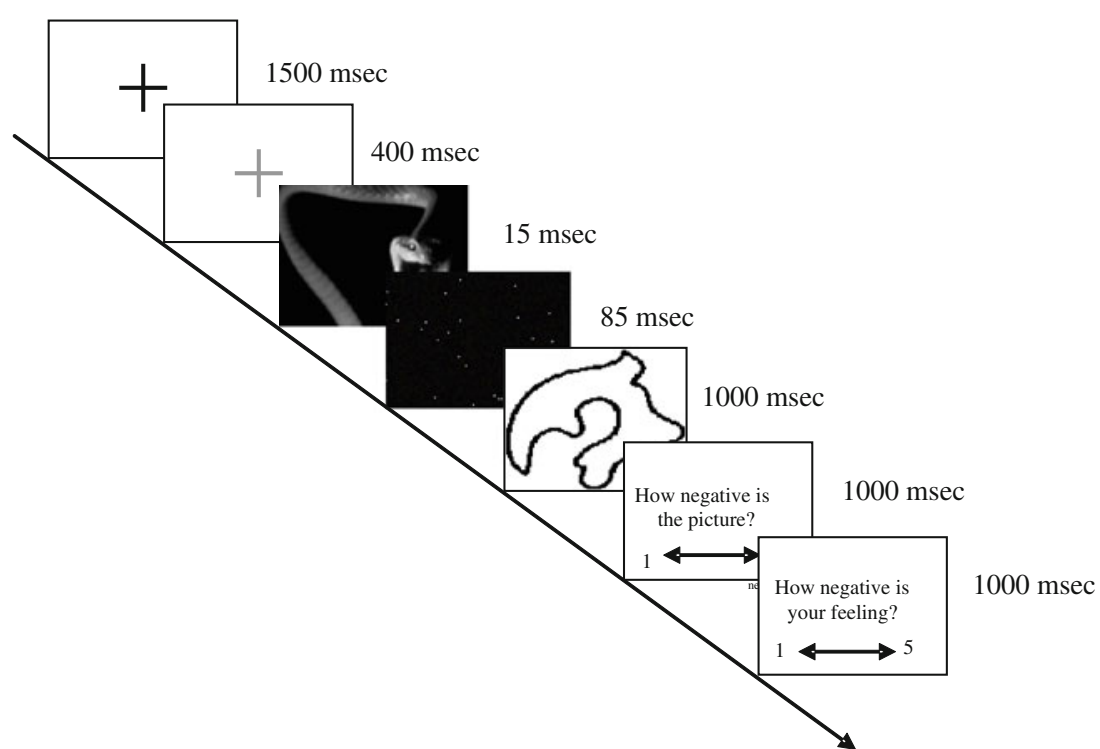

score was assessed using an unweighted average of the subscale scores, because high correlations have been shown between weighted and unweighted scores of the subscales (Byers, Bittner, \& Hill, 1989). The NASA Task Load Index scores were collected at the end of the experimental session.

Heart rate HR was recorded using an MP-100 psychophysiological monitoring system (BioPac Systems, Santa Barbara, CA) with a $35-\mathrm{Hz}$ low-pass filter and a $0.5-\mathrm{Hz}$ high-pass filter. For each participant, $\mathrm{Ag} / \mathrm{AgCl}$ electrodes filled with isotonic $\mathrm{NaCl}$ unibase electrolyte were attached to the right side of the neck and to the inner surface of the left forearm for recording an electrocardiogram. HR was measured continuously throughout the experimental session, and the data were analyzed offline using Acknowledge software (BioPac Systems, Santa Barbara, CA). Interbeat intervals were converted to HR as beats per minute per real-time epoch of $0.5 \mathrm{~s}$. We assessed two measures of the phasic responses of HR as follows. First, HR in two continuous epochs ( $1 \mathrm{~s})$ before and six continuous epochs ( $3 \mathrm{~s}$ ) after onset of a prime stimulus was analyzed on each trial. This analysis provided a detailed profile of the temporal change of HR, which was time-locked to presentation of the prime stimulus. Next, the change of HR was calculated by subtracting $\mathrm{HR}$ in the prestimulus epoch $(0.5 \mathrm{~s})$ from an average of HR in the two continuous postprime-stimulus epochs ( $1 \mathrm{~s}$ ) on each trial. This analysis provided an index of a local change of HR that reflected an emotional response to a prime stimulus.

\section{Data analysis}

All analyses were carried out with IBM SPSS Statistics 19. We conducted an analysis of variance (ANOVA) for each index. The Huynh-Feldt epsilon correction factor was used where appropriate. When a significant interaction effect or a significant main effect was found in an ANOVA, post hoc analyses using the Bonferroni correction were conducted to examine which combinations of data were significantly different. The statistical significance level was automatically adjusted to $p<.05$ by IBM SPSS Statistics 19 . Measures of effect size (partial eta-squared) were also obtained automatically using IBM SPSS Statistics 19 for all analyses where appropriate.

\section{Results}

For the overall scores of the NASA Task Load Index, we conducted an ANOVA with group (control, cognitive task, noncognitive task) as a between-participants factor. A significant main effect of group was shown, $F(2,42)=64.36$, $p<.01$, partial $\eta^{2}=.32$. Significant differences were found between the control group and the other two groups (cognitive task and noncognitive task; $p<.01$, respectively). While the cognitive task and noncognitive task groups showed higher scores of task load than did the control group, there was no significant difference in scores between the cognitive and noncognitive task groups (see Fig. 2). Then, to examine the effects of cognitive and noncognitive tasks on subscales of the NASA Task Load Index, we conducted an ANOVA with group (control, cognitive task, noncognitive task) as a between-participants factor and subscale (mental demands, physical demands, temporal demands, performance, effort, frustration) as a repeated measure, following a recommendation by Hart and Staveland (1988). A significant interaction between group and subscale was shown, $F(10,285)=13.68, p<.01$, partial $\eta^{2}=0.12$. In the cognitive task, scores of performance and effort were 


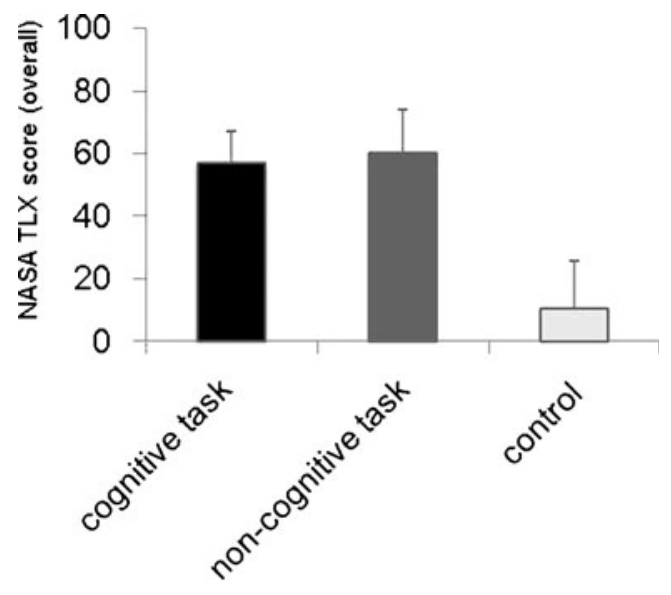

Fig. 2 Overall workload score of the NASA Task Load Index for each task. Error bars indicate standard errors

significantly higher than those of physical demand and temporal demand. In the noncognitive task, scores of physical demand and effort were significantly higher than those of temporal demand. In the control group, there was no significant difference among scores of subscales. Furthermore, scores in the cognitive and noncognitive task groups were significantly higher than those in the control group in all subscales. Scores of physical demand in the noncognitive task were significantly higher than those in the cognitive task (see Fig. 3). These results indicated that the mental demand and effort for each task were well controlled.

Data from the forced choice test of recognition of prime stimuli indicated that the subliminal presentation procedures were successful. The recognition ratio (i.e., the number of correct recognitions of the prime divided by the total number

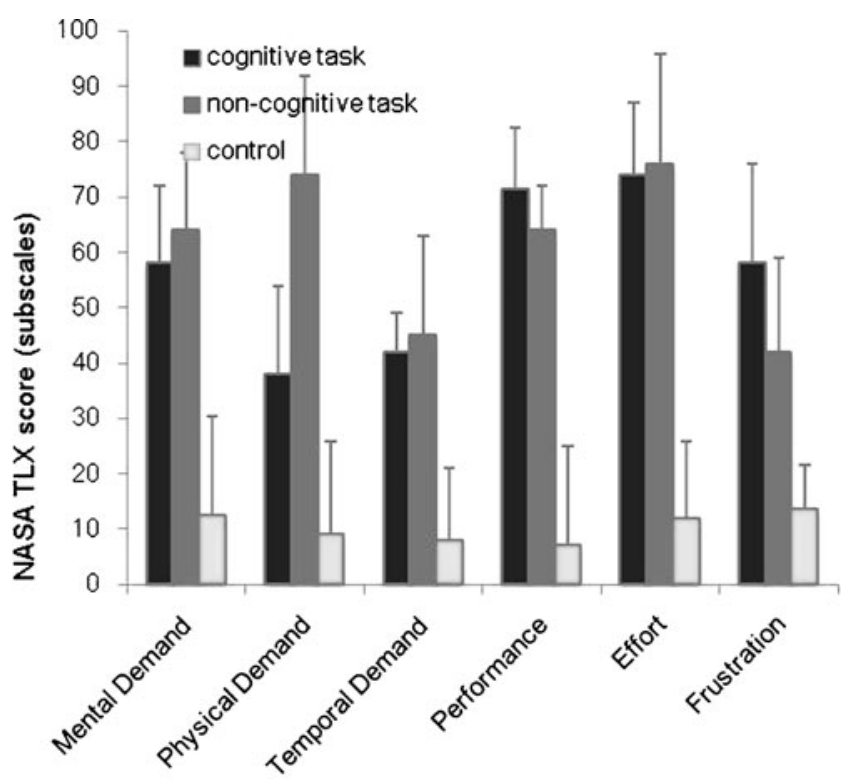

Fig. 3 Workload scores for each subscale of the NASA Task Load Index scores for each task. Error bars indicate standard errors of recognition judgments made) was $.52(S D=.13)$, which is not significantly different from the chance expectation of .50, using a chi-square test at the individual level and using a paired $t$-test at the group level. For the paired $t$-test at the group level, the recognition ratio did not differ as a function of the type of prime (negative, neutral).

Regarding the PANAS scores, which indicate participants' negative feelings, we conducted an ANOVA with group (control, cognitive task, noncognitive task) as a between-participants factor and stage (postbaseline, postcognitive-task, post-emotional-task, postrecovery) as a repeated measure. A significant interaction between group and stage was found, $F(6,171)=9.00, p<.01$, partial $\eta^{2}=.23$. The PANAS scores after the emotional task were significantly higher than those at the other stages in the control $(p<.01)$ and noncognitive task $(p<.05)$ groups. This result showed that the emotional task successfully caused negative emotions, as we expected. In the cognitive task group, there were no significant differences among stages. Moreover, after the emotional task, the score in the control group was significantly higher than that in the cognitive task group $(p<.01$; see Fig. 4).

For the negativity of target stimuli, we conducted an ANOVA with group (control, cognitive task, noncognitive task) as a between-participants factor and prime (negative, neutral) as a repeated measure. A significant interaction between group and prime was shown for the unpleasantness of target stimuli, $F(2,57)=8.74, p<.01$, partial $\eta^{2}=.21$. The score for negative primes was significantly higher than that for neutral primes in the control group $(p<.01$; see Fig. 5), but not in the cognitive and noncognitive task groups. There was no significant interaction, $F(2,57)=$ 2.85 , n.s., partial $\eta^{2}=.18$, and no significant main effects [prime, $F(1,57)=1.68$, n.s. , partial $\eta^{2}=.15$; group, $F(2,57)=$ 2.12, n.s., partial $\eta^{2}=.17$ ] for the negativity of participants' feelings (see Fig. 6).

To assess phasic responses of $\mathrm{HR}$, we conducted an ANOVA with epoch $(-1-0.5,-0.5-0,0-0.5,0.5-1,1-1.5$,

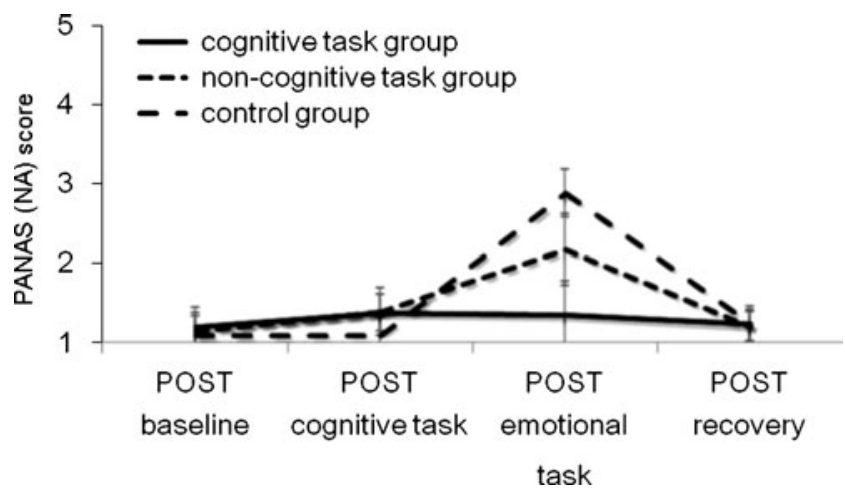

Fig. 4 Self-reports of negative emotion at pre-/post-executive-function task, postemotional task, and postrecovery for the each group. Error bars indicate standard deviations 


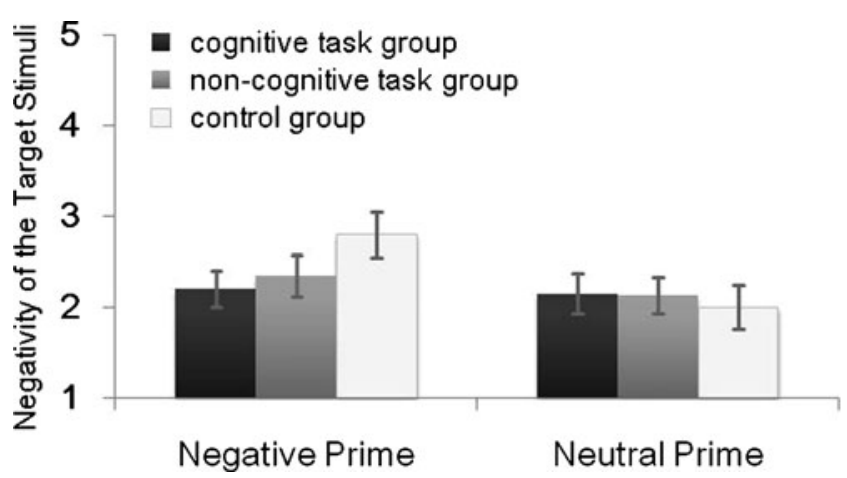

Fig. 5 Mean ratings of reported negativity of the target stimuli for group by prime. Error bars indicate standard deviations

1.5-2.0, 2.0-2.5, and 2.5-3.0 s) as a repeated measure, group (control, cognitive task, noncognitive task) as a between-participants factor, and prime (negative, neutral) as a repeated measure. A significant second-order interaction among epoch, group, and prime was shown, $F(14,399)=3.93$, $p<.01$, partial $\eta^{2}=.24$ (see Fig. 7). In the negative prime condition, HR in the control and noncognitive task groups was maintained at a significantly higher level in continuous epochs $0.5 \mathrm{~s}$ after onset of a prime, as compared with HR in the first epoch after onset of the prime $(0-0.5 \mathrm{~s} ; p<.05)$. In the neutral prime condition, HR in the noncognitive task group was maintained at a significantly higher level during a period from 0.5 to $2.0 \mathrm{~s}$ after onset of a prime, as compared with HR in the first epoch after onset of the prime $(0-0.5 \mathrm{~s} ; p<.05)$. In the cognitive task group, there was no significant difference among epochs in both prime conditions. Significant differences between HR for negative primes and HR for neutral primes were seen at epoch 1.0-2.0 s after onset of a prime in the control group $(p<.05)$. A significant difference between HR in the control group and HR in the cognitive task group was shown $0.5 \mathrm{~s}$ after onset of a prime in the negative prime condition $(p<.05)$. A significant difference between HR in the cognitive task group and HR in the noncognitive task group was shown at the epoch $1.0-1.5 \mathrm{sec}$ after onset of a prime in the negative prime conditions $(p<.05)$. There was no significant

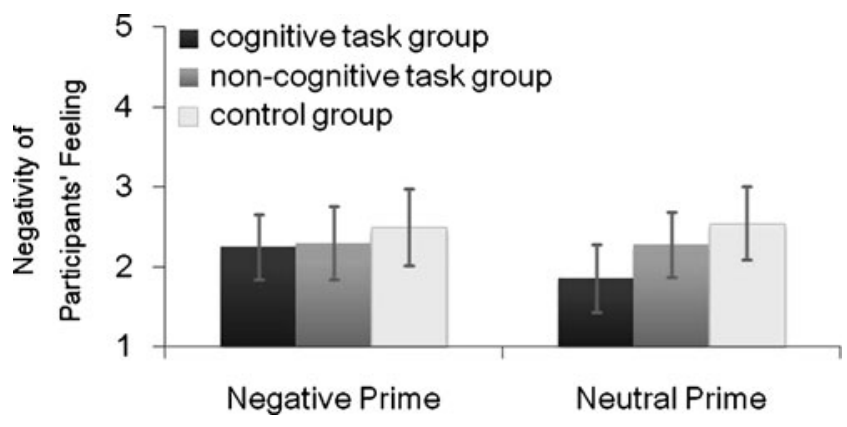

Fig. 6 Mean ratings of reported participants' feelings for group by prime. Error bars indicate standard deviations difference between HR in the noncognitive task group and HR in the control group in all epochs.

Then we conducted an ANOVA on local changes of HR by prime stimuli with group (control, cognitive task, noncognitive task) as a between-participants factor and prime (negative, neutral) as a repeated measure. A significant interaction between group and prime was shown, $F(2,57)=11.28, p<.01$, partial $\eta^{2}=.21$. In the negative prime condition in the cognitive task group, HR significantly decreased, as compared with the other groups $(p<.05)$. Within groups, HR significantly decreased in the negative prime condition, as compared with the neutral prime condition, in the cognitive task group $(p<.01)$, whereas HR significantly increased in the negative prime condition, as compared with the neutral prime condition, in the control group $(p<.05)$. In the noncognitive task group, changes of HR were not different between the negative prime condition and the neutral prime condition (see Fig. 8).

\section{Discussion}

In the control group of this study, subliminal presentation of a negative affective picture (prime) shifted subsequent affective evaluation of a novel and neutral figure (target) in the direction affectively congruent with the prime stimulus. This result is consistent with those in previous studies (e.g., Payne, Cheng, Govorun, \& Stewart, 2005) and suggested the validity of the experimental paradigm in this study. On the other hand, in the cognitive task group, as we expected, subliminal presentation of a negative affective picture did not shift the affective evaluation of a target. This result supported the idea that the prior performance of a cognitive task can inhibit the subliminal affective priming effect. In addition, participants in the cognitive task group reported significantly lower negative affects after the emotional task than did the control and noncognitive task groups. Furthermore, the cognitive task group showed significantly reduced responses in phasic HR, as compared with the control group and the noncognitive task group, whereas there was no significant difference of phasic HR responses between the control group and the noncognitive task group. These results suggested that prior cognitive activity attenuated physiological responses to subsequently presented emotional stimuli. These results of self-report and HR data successfully replicated the findings of Iida et al. (2011) and expanded them by showing that attenuation of emotional responses can occur even when emotional stimuli were presented subliminally. Taken together, the present study provides further evidence that cognitive activity is a critical factor in the implicit attenuation of subsequent emotions. Most important, going beyond previous studies, we showed that cognitive activity can affect the bottom-up appraisal of emotional stimuli. 
Fig. 7 Temporal sequence of heart rate after onset of the prime stimulus

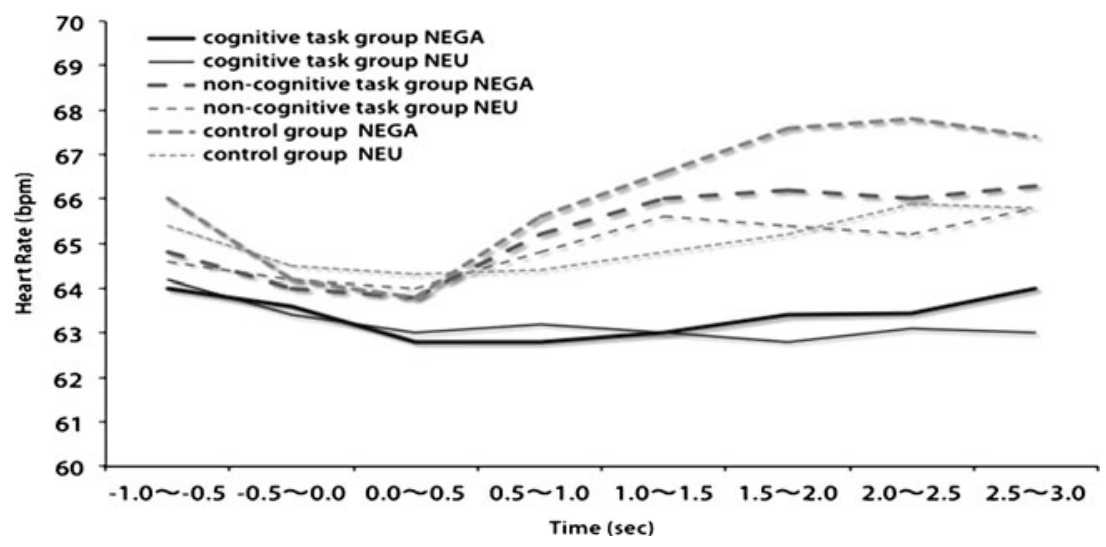

In general, a triphasic pattern of HR responses to novel stimuli including emotional stimuli is observed - that is, an initial deceleration, then an acceleration, which is followed by a second deceleration (Cook \& Turpin, 1997; Graham, 1992; Lang et al., 1998; Murakami, Matsunaga, \& Ohira, 2010). Especially when an emotional stimulus is presented briefly or subliminally, usually the initial deceleration of HR is reduced and the acceleration of HR becomes dominant (Codispoti, Bradley, \& Lang, 2001; Critchley et al., 2005; Jönsson \& Sonnby-Borgström, 2003). This acceleration has been thought to be caused by cardio-sympathetic activity and to reflect the defensive reflex to a stimulus and the rejection of information about the stimulus (Cook \& Turpin, 1997). Thus, transient increases of HR after onset of primes observed in the control group and the noncognitive task group suggested that the primes presented subliminally could be detected unconsciously and could cause emotional responses in participants. Lack of this phasic HR response to primes observed in the cognitive task group suggested that prior performance of a cognitive task might attenuate emotional processes to stimuli even at a very early stage.

One of the most significant questions here is how prior cognitive activity attenuates subsequent emotional responses. Iida et al. (2011) suggested that performance of a cognitive

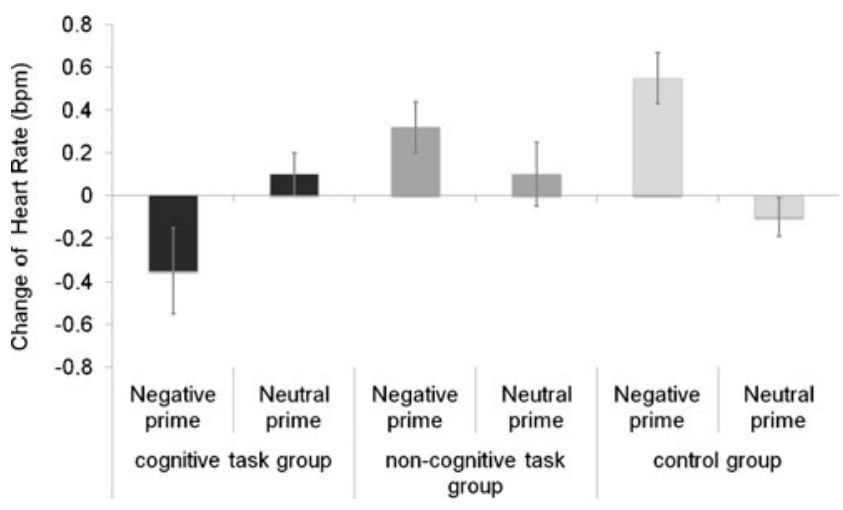

Fig. 8 Change of heart rate by prime and group. Error bars indicate standard deviations task can continuously boost executive function for a certain period and potentially modulate the way that participants process subsequent emotional stimuli. Some previous studies showed that the prior performance of cognitive tasks modulated cognitive strategies and the underlying patterns of neural activation in subsequent cognitive tasks (e.g., Browning et al., 2010). Supporting this notion, increased brain activity in several prefrontal and parietal regions following a single session of cognitive training has been shown (Browning, Holmes, Murphy, Goodwin, \& Harmer, 2010; Olesen, Westerberg, \& Klingberg, 2004). These brain regions have been implicated in emotion regulation, cognitionemotion interactions, and top-down direction of attention in response to negative emotional stimuli (Beer, Heerey, Keltner, Scabini, \& Knight, 2003; Davidson, 2002; Elliott, Dolan, \& Frith, 2000; Gottfried, O'Doherty, \& Dolan, 2003; Hamann, Ely, Hoffman, \& Kilts, 2002; Lieberman, 2000; Ohira et al., 2006; Rolls, 2000; for a review, see Mauss, Bunge, \& Gross, 2007). Additionally, even if the emotional stimuli were presented subliminally, the activity of the ventrolateral prefrontal cortex (PFC) automatically occurred to regulate emotional responses caused by the amygdala (Nomura et al., 2004). Hence, enduring activation of the PFC caused by engagement in a prior cognitive task might unintentionally affect the subsequent emotional processing at its earliest stage and attenuate emotional responses, even when an emotional stimulus is presented subliminally. We should recognize that the above-described mechanisms possibly underlying the present results are merely speculative. Neuroimaging studies using functional magnetic resonance imaging and electroencephalogram are needed to clarify this possibility.

We should notice the differences in the effects of the cognitive task and the noncognitive (handgrip-squeezing) task on the emotional task. The noncognitive task did not affect subjective affective states and HR responses in the emotional task but reduced the subliminal affective priming effect in evaluation of target stimuli as the cognitive task. To 
understand this result, it might be helpful to recognize that the subliminal affective priming effect has been thought to consist of two automatic processes: emotion elicitation and misattribution (e.g., Schwarz \& Clore, 1988; Zajonc, 1980). That is to say, if there is interference in either process, the subliminal affective priming effect is diminished. In the cognitive task group, the automatic emotion elicitation was possibly attenuated as described above. On the other hand, since the noncognitive task group showed similar subjective affective states and HR responses in the emotional task with the control group, it can be considered that the noncognitive task did not interfere with emotion elicitation. Thus, the lack of the affective priming effect in this group should be attributed to interference in the process of misattribution. One possible explanation for this speculation might be that engagement in the noncognitive task elicited the motivation of self-control, which was carried over into the subsequent emotional task. One of the ingredients of self-control is motivation to achieve a goal or to meet a standard (Baumeister $\&$ Vohs, 2007). Some previous studies have shown that this motivation could be carried over into a subsequent self-control task and could improve task performance in the subsequent task (e.g., Martijn et al., 2007; Webb \& Sheeran, 2003). Indeed, the handgrip-squeezing task we used as a noncognitive task in this study has been thought to be a typical task requiring self-control (e.g., Muraven \& Baumeister, 2000; Muraven, Tice, \& Baumeister, 1998). In the affective priming task in this study, we asked participants to evaluate the affective negativity of target stimuli. Since all the target stimuli were emotionally neutral, if the motivated participants tried to engage in this task, the affective influence could be canceled, and the affective priming effect could be diminished. Since emotion elicitation reflected in the subjective affective states and HR is a fully automatic process, participants could not attenuate these emotional responses even with the enhanced self-control motivation.

In spite of remaining questions about the underlying mechanisms, this study showed some initial evidence that engaging in cognitive activity can attenuate psychological and physiological responses even to emotional stimuli that are subliminally presented.

Author Note The authors are very grateful to Naho Ichikawa, Stanford University, for discussion about the design of this study and to Takahiro Osumi, Keio University, for advice on analyses on autonomic responses. The authors would like to extend their thanks to the editor and two anonymous reviewers for their carefully considered feedback on a previous draft of this manuscript.

\section{Sources of financial support None}

\section{Conflicts of interest Absent}

\section{Appendix}

The numbers of the IAPS pictures which were used in this study were the following: neutral $(1510,1560,1660,1670$, 2020, 2190, 2210, 2215, 2280, 2372, 2383, 2393, 2487, 2491, 2520, 2570, 2600, 2635, 2745.1, 4534, 5500, 5520, 5534, 7004, 7006, 7009, 7010, 7020, 7025, 7030, 7035, 7036, 7037, 7038, 7041, 7050, 7095, 7100, 7179, 7183, 7205, 7217, 7224, 7235, 7238, 7491, 7493, 7496, 7500, $7550,7560,7595,7705,7710,7950,8211,8250,8490$, $9070,9156)$ and unpleasant $(1040,1050,1070,1200$, $1201,1220,1274,1300,1930,2095,2683,2694,2710$, $3500,6211,6212,6213,6244,6250,6260,6311,6312$, $6315,6370,6510,6530,6560,6570.1,6821,6830,6831$, 6834, 6838, 6940, 7359, 7380, 8230, 8480, 9050, 9140, 9250, 9340, 9342, 9404, 9409, 9421, 9435, 9440, 9470, 9471, 9480, 9530, 9560, 9584, 9592, 9611, 9630, 9635.1, 9830, 9911).

\section{References}

Baddeley, A. D. (2003). Working memory: Looking back and looking forward. Nature Reviews Neuroscience, 4, 829-839.

Barrett, L. F., Ochsner, K. N., \& Gross, J. J. (2007). On the automaticity of emotion. In J. Bargh (Ed.), Social psychology and the unconscious: The automaticity of higher mental processes (pp. 173-217). New York: Psychology Press.

Baumeister, R. F., \& Vohs, K. D. (2007). Self-regulation, ego depletion, and motivation. Social and Personality Psychology Compass, 1, 114.

Beer, J. S., Heerey, E. A., Keltner, D., Scabini, D., \& Knight, R. T. (2003). The regulatory function of self-conscious emotion: Insights from patients with orbitofrontal damage. Journal of Personality and Social Psychology, 85, 594-604.

Berridge, K. C., \& Winkielman, P. (2003). What is an unconscious emotion: The case for unconscious "liking". Cognition and Emotion, $17,181-211$.

Browning, M., Holmes, E. A., Murphy, S. E., Goodwin, G. M., \& Harmer, C. J. (2010). Lateral prefrontal cortex mediates the cognitive modification of attentional bias. Biological Psychiatry, 67, 919-925.

Byers, J. C., Bittner, A. C., \& Hill, S. G. (1989). Traditional and raw task load index (TLX) correlations: Are paired comparisons necessary? In A. Mital (Ed.), Advances in industrial ergonomics and safety (Vol. 1, pp. 481-485). London: Taylor \& Francis.

Codispoti, M., Bradley, M. M., \& Lang, P. J. (2001). Affective reactions to briefly presented pictures. Psychophysiology, 38, 474478.

Cook, E. W., \& Turpin, G. (1997). Differentiating orienting, startle, and defense responses: The role of affect and its implications for psychopathology. In P. J. Lang, R. F. Simons, \& M. Balaban (Eds.), Attention and orienting: Sensory and motivational processes (pp. 137-164). Mahwah, NJ: Erlbaum.

Critchley, H. D., Rotshtein, P., Nagai, Y., O'Doherty, J., Mathias, C. J., \& Dolan, R. J. (2005). Activity in the human brain predicting differential heart rate responses to emotional facial expressions. NeuroImage, 24, 751-762.

Davidson, R. J. (2002). Anxiety and affective style: Role of prefrontal cortex and amygdala. Biological Psychiatry, 51, 68-80. 
Elliott, R., Dolan, R. J., \& Frith, C. D. (2000). Dissociable functions in the medial and lateral orbitofrontal cortex: Evidence from human neuroimaging studies. Cerebral Cortex, 10, 308-317.

Endo, N., Saiki, J., \& Saito, H. (2001). Determinants of occurrence of negative priming for novel shapes with matching paradigm. Japanese Journal of Psychology, 72, 204-212.

Erk, S., Abler, B., \& Walter, H. (2006). Cognitive modulation of emotion anticipation. European Journal of Neuroscience, 24, 1227-1236.

Gottfried, J. A., O'Doherty, J., \& Dolan, R. J. (2003). Encoding predictive reward value in human amygdala and orbitofrontal cortex. Science, 301, 1104-1107.

Graham, F. K. (1992). Attention: The heartbeat, the blink, and the brain. In B. A. Campbell, H. Hayne, \& R. Richardson (Eds.), Attention and information processing in infants and adults: Perspectives from human and animal research (pp. 3-29). Hillsdale, NJ: Erlbaum.

Haga, S., \& Mizukami, N. (1996). Japanese version of NASA Task Load Index: Sensitivity of its workload score to difficulty of three different laboratory tasks. Japanese Journal of Ergonomics, 32, 71-79.

Hamann, S. B., Ely, T. D., Hoffman, J. M., \& Kilts, C. D. (2002). Ecstasy and agony: Activation of human amygdala in positive and negative emotion. Psychological Science, 13, 135-141.

Hariri, A. R., Mattay, V. S., Tessitore, A., Fera, F., \& Weinberger, D. R. (2003). Neocortical modulation of the amygdala response to fearful stimuli. Biological Psychiatry, 53, 494-501.

Hart, S. G., \& Staveland, L. E. (1988). Development of NASA-TLX (Task Load Index): Results of empirical and theoretical research. In P. A. Hancock \& N. Meshkati (Eds.), Human mental workload (pp. 139-183). Amsterdam: Elsevier.

Iida, S., Nakao, T., \& Ohira, H. (2011). Implicit attenuation of a subsequent emotion by cognitive activity. Cognitive, Affective, \& Behavioral Neuroscience, 11, 476-484. doi:10.3758/s13415-011-0045-y

Jönsson, P., \& Sonnby-Borgström, M. (2003). The effects of pictures of emotional faces on tonic and phasic autonomic cardiac control in women and men. Biological Psychology, 62, 157-173.

Jostmann, N., Koole, S. L., Van der Wulp, N., \& Fockenberg, D. (2005). Subliminal affect regulation: The moderating role of action versus state orientation. European Psychologist, 10, 209-217.

Lang, P. J., Bradley, M. M., \& Cuthbert, B. N. (1999). The International Affective Pictures System (IAPS): Technical manual and affective ratings. Gainsville: University of Florida.

Lang, P. J., Bradley, M. M., Fitzsimmons, J. R., Cuthbert, B. N., Scott, J. D., Moulder, B., \& Nangia, V. (1998). Emotional arousal and activation of the visual cortex: An fMRI analysis. Psychophysiology, $35,199-210$.

Lieberman, M. D. (2000). Intuition: A social cognitive neuroscience approach. Psychological Bulletin, 126, 109-137.

Liberzon, I., Taylor, S. F., Fig, L. M., Decker, L. R., Koeppe, R. A., \& Minoshima, S. (2000). Limbic activation and psychophysiologic responses to aversive visual stimuli: Interaction with cognitive task. Neuropsychopharmacology, 23, 508-516.

Martijn, C., Alberts, H. J. E. M., Merckelbach, H., Havermans, R., Huijts, A., \& de Vries, N. K. (2007). Overcoming ego depletion: The influence of exemplar priming on self-control performance. European Journal of Social Psychology, 37, 231-238.

Mauss, I. B., Bunge, S. A., \& Gross, J. J. (2007). Automatic emotion regulation. Social and Personality Psychology Compass, 1, 146-167.

McRae, K., Misra, S., Prasad, A. K., Pereira, S. C., \& Gross, J. J. (2011). Bottom-up and top-down emotion generation: Implications for emotion regulation. Social Cognitive and Affective Neuroscience. doi:10.1093/scan/nsq103

Monk, C. S., McClure, E. B., Nelson, E. E., Zarahn, E., Bilder, R. M., Leibenluft, E., ... Pine, D. S. (2003). Adolescent immaturity in attention-related brain engagement to emotional facial expressions. NeuroImage, 20, 420-428.

Murakami, H., Matsunaga, M., \& Ohira, H. (2010). Phasic heart rate responses for anticipated threat situations. International Journal of Psychophysiology, 77, 21-25.

Muraven, M. R., \& Baumeister, R. F. (2000). Self-regulation and depletion of limited resources: Does self-control resemble a muscle? Psychological Bulletin, 126, 247-259.

Muraven, M., Tice, D. M., \& Baumeister, R. F. (1998). Self-control as limited resource: Regulatory depletion patterns. Journal of Personality and Social Psychology, 74, 774-789.

Nomura, M., Ohira, H., Haneda, K., Iidaka, T., Sadato, N., Okada, T., \& Yonekura, Y. (2004). Functional association of the amygdala and ventral prefrontal cortex during cognitive evaluation of facial expressions primed by masked angry faces: An event-related fMRI study. NeuroImage, 21, 352-363.

Ochsner, K. N., \& Gross, J. J. (2007). The neural architecture of emotion regulation. In J. J. Gross (Ed.), Handbook of emotion regulation (pp. 87-109). New York: Guilford.

Ochsner, K. N., Ray, R. R., Cooper, J. C., Robertson, E. R., Chopra, S., Gabrieli, J. D. E., \& Gross, J. J. (2004). For better or for worse: Neural systems supporting the cognitive down- and up-regulation of negative emotion. NeuroImage, 23, 483-499.

Ohira, H., Nomura, M., Ichikawa, N., Isowa, T., Iidaka, T., Sato, A., ... Yamada, J. (2006). Association of neural and physiological responses during voluntary emotion suppression. NeuroImage, 29, 721-733.

Olesen, P. J., Westerberg, H., \& Klingberg, T. (2004). Increased prefrontal and parietal activity after training of working memory. Nature Neuroscience, 7, 75-79.

Payne, B. K., Cheng, C. M., Govorun, O., \& Stewart, B. D. (2005). An inkblot for attitudes: Affect misattribution as implicit measurement. Journal of Personality and Social Psychology, 89, 277-293.

Rolls, E. T. (2000). The orbitofrontal cortex and reward. Cerebral Cortex, 10, 284-294.

Sato, A., \& Yasuda, A. (2001). Development of the Japanese version of Positive and Negative Affect Schedule (PANAS) scales. Japanese Journal of Personality, 9, 138-139.

Schwarz, N., \& Clore, G. L. (1988). How do I feel about it? Informative functions of affective states. In K. Fiedler \& J. Forgas (Eds.), Affect, cognition, and social behavior (pp. 44-62). Toronto, Canada: Hogrefe.

Wallbott, H. G., \& Ricci-Bitti, P. (1993). Decoders' processing of emotional facial expression - a top-down or bottom-up mechanism? European Journal of Social Psychology, 23, 427-443.

Webb, T. L., \& Sheeran, P. (2003). Can implementation intentions help to overcome ego-depletion? Journal of Experimental Social Psychology, 39, 279-286.

Zajonc, R. B. (1980). Feeling and thinking: Preferences need no inferences. American Psychologist, 35, 117-123.

Zajonc, R. B. (2000). Feeling and thinking: Closing the debate over the independence of affect. In J. P. Forgas (Ed.), Feeling and thinking: The role of affect in social cognition (pp. 31-58). Cambridge: Cambridge University Press. 